\title{
Ulam Stability in Real Inner-Product Spaces
}

\author{
Alexandra MĂduță AND Bianca Moşneguțu*
}

ABSTRACT. Roughly speaking an equation is called Ulam stable if near each approximate solution of the equation there exists an exact solution. In this paper, we prove that Cauchy-Schwarz equation, Orthogonality equation and Gram equation are Ulam stable.

Keywords: Ulam stability, inner-product space.

2010 Mathematics Subject Classification: 39B72, 39B82.

\section{INTRODUCTION}

This paper is concerned with the Ulam stability of some classical equations arising in the context of inner-product spaces. For the general notion of Ulam stability see, e.q., [1]. Roughly speaking an equation is called Ulam stable if near every approximate solution there exists an exact solution; the precise meaning in each case presented in this paper is described in three theorems. Related results can be found in [2,3,4]. See also [5] for some inequalities in inner product spaces.

\section{THE CAUCHY-SCHWARZ EQUATION}

Let $(V,(\cdot \mid \cdot))$ be a real inner-product space. Consider the Cauchy-Schwarz equation, i.e.,

$$
\|x\|^{2}\|y\|^{2}-(x \mid y)^{2}=0 \text {. }
$$

The set of its solutions is

$$
S=\left\{(x, y) \in V^{2}: x, y \text { are linearly dependent vectors }\right\} .
$$

Theorem 2.1. Let $\varepsilon>0$ and $(u, v) \in V^{2}$ an approximate solution of (2.1), i.e.,

$$
\|u\|^{2}\|v\|^{2}-(u \mid v)^{2} \leq \varepsilon .
$$

Then there exists an exact solution $(x, y) \in S$ such that

$$
\|u-x\|^{2}+\|v-y\|^{2} \leq \sqrt{\varepsilon} .
$$

Proof. If $u$ and $v$ are linearly dependent, then it suffices to take $x=u, y=v$. So, let $u$ and $v$ be linearly independent. Then $u \neq 0, v \neq 0$; suppose that $\|v\| \leq\|u\|$ and let $w_{t}:=u+t v, t \in \mathbb{R}$. Then $w_{t} \neq 0, t \in \mathbb{R}$; let $z_{t}:=w_{t} /\left\|w_{t}\right\|$ and $W_{t}:=\operatorname{Span}\left\{z_{t}\right\}$. Let $x_{t}:=p r_{W_{t}} u$ and $y_{t}:=p r_{W_{t}} v$ be the orthogonal projections of $u$, respectively $v$, on $W_{t}$. Then $x_{t}$ and $y_{t}$ are linearly dependent 
vectors, i.e., $\left(x_{t}, y_{t}\right) \in V^{2}$ is a solution of (2.1). Moreover, $\left\|u-x_{t}\right\|^{2}+\left\|v-y_{t}\right\|^{2}=\|u\|^{2}-\left(u \mid z_{t}\right)^{2}+$ $\|v\|^{2}-\left(v \mid z_{t}\right)^{2}$. Let $a:=(v \mid v), b:=(u \mid v), c:=(u \mid u)$. Then

$$
a c-b^{2}>0 ; 0<a \leq c .
$$

We have

$$
\left\|u-x_{t}\right\|^{2}+\left\|v-y_{t}\right\|^{2}=c+a-\left(u \mid z_{t}\right)^{2}-\left(v \mid z_{t}\right)^{2}
$$

and

so that

$$
s:=s(t):=\left(u \mid z_{t}\right)^{2}+\left(v \mid z_{t}\right)^{2}=\frac{\left(u \mid w_{t}\right)^{2}+\left(v \mid w_{t}\right)^{2}}{\left\|w_{t}\right\|^{2}}=\frac{(c+t b)^{2}+(b+t a)^{2}}{\left\|w_{t}\right\|^{2}},
$$

It follows that

$$
s=\frac{\left(a^{2}+b^{2}\right) t^{2}+2 b(a+c) t+b^{2}+c^{2}}{a t^{2}+2 b t+c} .
$$

which entails

$$
\left(a^{2}+b^{2}-a s\right) t^{2}+2 b(a+c-s) t+b^{2}+c^{2}-c s=0,
$$

From (2.5) and (2.7), we deduce

$$
s^{2}-(a+c) s+a c-b^{2} \leq 0 .
$$

Let $s_{1}$ and $s_{2}$ be the roots of the corresponding equation, i.e.,

$$
s_{1}=\frac{a+c-\sqrt{(a-c)^{2}+4 b^{2}}}{2}, s_{2}=\frac{a+c+\sqrt{(a-c)^{2}+4 b^{2}}}{2} .
$$

Then $s_{1} \leq s(t) \leq s_{2}$ for all $t \in \mathbb{R}$. By using (2.5), it is easy to prove that there exists $\tau \in \mathbb{R}$ such that $s(\tau)=s_{2}$. Now,

$$
\begin{gathered}
\left\|u-x_{\tau}\right\|^{2}+\left\|v-x_{\tau}\right\|^{2}=a+c-s(\tau)=a+c-s_{2}=a+c-\frac{a+c+\sqrt{(a-c)^{2}+4 b^{2}}}{2}= \\
=\frac{a+c-\sqrt{(a-c)^{2}+4 b^{2}}}{2} \leq \sqrt{a c-b^{2}}=\sqrt{\|u\|^{2}\|v\|^{2}-(u \mid v)^{2}} .
\end{gathered}
$$

Combined with (2.3), this gives (2.4) and the proof is finished.

\section{THE ORTHOGONALITY EQUATION}

Consider the orthogonality equation $(x \mid y)=0$.

Theorem 3.2. Let $\varepsilon>0$ and $(u, v) \in V^{2}$ such that $\|u\|=\|v\|=1$ and $|(u \mid v)| \leq \varepsilon$. Then, there exists $(x, y) \in V^{2}$ such that $\|x\|=\|y\|=1,(x \mid y)=0$ and

$$
\|u-x\|^{2}+\|v-y\|^{2} \leq(4-2 \sqrt{2}) \varepsilon .
$$

Proof. (i) Let $(u \mid v)>0$. Choose $w \in \operatorname{Span}\{u, v\},\|w\|=1,(w \mid u)=0$. Then $v=u \cos \alpha+w \sin \alpha$, for a suitable $\alpha \in\left[0, \frac{\Pi}{2}\right)$. Define $x_{t}:=u \cos t-w \sin t, y_{t}:=u \sin t+w \cos t, t \in \mathbb{R}$. Then $\left\|x_{t}\right\|=$ $1,\left\|y_{t}\right\|=1,\left(x_{t} \mid y_{t}\right)=0$, and $\left\|u-x_{t}\right\|^{2}+\left\|v-y_{t}\right\|^{2}=\|(1-\cos t) u+w \sin t\|^{2}+\|(\cos \alpha-\sin t) u+$ $(\sin \alpha-\cos t) w \|^{2}=(1-\cos t)^{2}+\sin ^{2} t+(\cos \alpha-\sin t)^{2}+(\sin \alpha-\cos t)^{2}=4-2((1+\sin \alpha) \cos t+$ $\cos \alpha \sin t)$. Clearly $(1+\sin \alpha) \cos t+\cos \alpha \sin t \leq \sqrt{2+2 \sin \alpha}, t \in \mathbb{R}$. Choose $\tau \in \mathbb{R}$ such that

$$
(1+\sin \alpha) \cos \tau+\cos \alpha \sin \tau=\sqrt{2+2 \sin \alpha} .
$$


Then

$$
\left\|u-x_{\tau}\right\|^{2}+\left\|v-y_{\tau}\right\|^{2}=4-2 \sqrt{2} \sqrt{1+\sin \alpha} .
$$

Now consider the function $f(\alpha)=(4-2 \sqrt{2}) \cos \alpha-4+2 \sqrt{2} \sqrt{1+\sin \alpha}, \alpha \in\left[0, \frac{\Pi}{2}\right]$. It is easy to verify that $f(0)=f\left(\frac{\Pi}{2}\right)=0$ and there exists $0<\beta<\frac{\Pi}{2}$ such that $f$ is increasing on $[0, \beta]$ and decreasing on $\left[\beta, \frac{\Pi}{2}\right]$. It follows that $f(\alpha) \geq 0, \alpha \in\left[0, \frac{\Pi}{2}\right]$; combined with (3.9), this yields

$$
\left\|u-x_{\tau}\right\|^{2}+\left\|v-y_{\tau}\right\|^{2} \leq(4-2 \sqrt{2}) \cos \alpha .
$$

On the other hand, $\cos \alpha=(u \mid v) \leq \varepsilon$, and so (3.8) is a consequence of (3.10).

(ii) If $(u \mid v)<0$, it suffices to use the proof of (i) with $v$ replaced by $-v$. Thus, the theorem is proved.

\section{THE GRAM EQUATION}

Denote by $G\left(u_{1}, \ldots, u_{m}\right)$ the Gram determinant of the vectors $u_{1}, \ldots, u_{m} \in V$. Let $v_{1}, \ldots, v_{n} \in V$ be linearly independent vectors. Consider the equation

$$
G\left(x, v_{1}, \ldots, v_{n}\right)=0 .
$$

Theorem 4.3. Let $\varepsilon>0$ and $u \in V$ such that

$$
G\left(u, v_{1}, \ldots, v_{n}\right) \leq \varepsilon .
$$

Then, there exists $x \in V$ which satisfy (4.11) and

$$
\|u-x\| \leq \frac{1}{\sqrt{G\left(v_{1}, \ldots, v_{n}\right)}} \sqrt{\varepsilon} .
$$

Proof. Let $W=\operatorname{Span}\left\{v_{1}, \ldots, v_{n}\right\}$ and $x:=p r_{W} u$. Then $x \in W$ and therefore it satisfies (4.11). Moreover,

$$
\|u-x\|=\sqrt{\frac{G\left(u, v_{1}, \ldots, v_{n}\right)}{G\left(v_{1}, \ldots, v_{n}\right)}} \leq \frac{1}{\sqrt{G\left(v_{1}, \ldots, v_{n}\right)}} \sqrt{\varepsilon} .
$$

\section{REFERENCES}

[1] J. Brzdęk, D. Popa, I. Raşa and Xu. B: Ulam stability of operators. Academic Press, London (2018).

[2] D. S. Marinescu, M. Monea and C. Mortici: Some characterizations of inner product spaces via some geometrical inequalities. Appl. Anal. Discrete Math. 11 (2017), 424-433.

[3] N. Minculete: Considerations about the several inequalities in an inner product space. Math. Inequalities 1 (2018), $155-161$.

[4] S. M. S. Nabavi: On mappings which approximately preserve angles. Aequationes Math. 92 (2018), 1079-1090.

[5] D. Popa, I. Raşa: Inequalities involving the inner product. JIPAM 8 (3) (2007), Article 86.

TECHNiCAL UNIVERSITY OF CLUJ-NAPOCA

DEPARTMENT OF MATHEMATICS

25, G. BARIŢiU STREET, 400027 CluJ-NAPOCA, ROMANiA

E-mail address: Alexandra.Măduţă@math.utcluj.ro

TECHNICAL UNIVERSITY OF CLUJ-NAPOCA

DEPARTMENT OF MATHEMATICS

25, G. BARIŢiU STREET, 400027 CluJ-NAPOCA, ROMANIA

ORCID: 0000-0002-7959-5638

E-mail address: Bianca.Moşneguţu@math.utcluj.ro 